\title{
Non-linear Frequency Modulated Signal Detection Based on Time-frequency Tiling
}

\author{
J. Wang, P.L. Shui \\ Institute of Electronic Engineering \\ Xidian University, Xi'an, 710071, P. R. China
}

\begin{abstract}
This paper proposes a novel time-frequency tiling based detector for detection of non-linear frequency modulated (NLFM) signal or polynomial phase signal (PPS). The method exploits the time-frequency tiling property of adaptive wavelet transiform, and is practically realizable. In the paper, the unitary and linear phase wavelet basis function together with joint one order linear approaching procedure is presented to estimate the signals trace line in time-frequency plane. Additionally, the Radon-Wigner (RWT) transform is exploited in subband outputs of time-frequency tiling to achieve low computational complexity implementation. Simulation results confirm the efficacy of the method.
\end{abstract}

Keywords-Detection, NLFM signal, time-frequency tiling

\section{I.INTRODUCTION}

Time-frequency analysis has become an important technique in analyzing wideband and nonstationary signals in various applications including biomedical signal analysis, FM radio communications, speech signal analysis and inverse synthetic radar imaging[1]. Non-linear frequency modulated(NFLM) signal, or polynomial phase signal (PPS) is a widely used model that linear frequency modulated (LFM) signal, or chirp signal, is a special case follows the model. Many efforts have been taken for its detection based on the practical allowable approximations. Wigner distribution (WD) has been found to be very useful in analyzing and detecting LFM signal [2]. The developed fast algorithm can be implemented by computing the line integral of WD of the signal along all the lines in the time-frequency plane, which is known as de-chirping algorithm, or Radon-Wigner transform (RWT) [3]. Other algorithms, such as short time Fourier transform, wavelet transform, and Radon-ambiguity transform $[4,5]$ have also been developed for the detection of the LFM signal. For the detection of NFLM signal, due to its non-linear frequency variation property, the performance of WD or RWT based detector will be decreased. Thus new method should be developed to combat the performance degradation when WD or RWT is used.

In this paper, a novel long-term coherent integration method is proposed for the detection of weak NLFM signal embedded in noise. The framework of the proposed detector is based on adaptive wavelet transform (AWT) of the signals. The detector is to search the signal trace line in time-frequency plane and conducts the coherent integration along the non-linear time-frequency trace line. It is known that AWT can be used to describe the non-linear and non-stationary characteristics of received signals, therefore, we use AWT to analyze the non-linear frequency variation of NLFM signals. By making use of AWT, the signal trace line in time-frequency plane can be decomposed into different local time-frequency tiling under certain criterion. The implementation of the proposed detector is based on the coherent integration in each tilings for which the frequency variation rate can be approximated to constant. Together with fast de-chirping algorithm used in each tiling, the implementation of the detector is equivalent to tracking the signal non-linear time-frequency trace line by linear tiling approximation, so that the detection performance and the output signal to noise ratio can be improved compared to the traditional RWD based detector which considered the variation of frequency is linear.

For the detection of weak NLFM signal, the AWT based detector is a sub-optimal one in case of maximum likelihood optimization. Using multi-resolution processing reduces the multi-dimensional optimization estimation of a non-linear to some related one-order linear approximations while hold acceptable computation complexity.

\section{II.Complex Orthogonal And Linear Phase Wavelet BASIS}

Wavelet analysis is a local time-frequency method which can provide detailed description for non-stationary characteristics of signal, multi-scale and multi-resolution capabilities enlarge the freedom of signal analyzing, improved AWT method provides an optimal representation of signal for scale space approaching and filtering. However, different basis has different resolution capability. It is important for us to choose a wavelet basis that is favorable for signal processing. For coherent integration detection of complex valued NLFM signal, we introduce here a complex symmetric orthogonal wavelet basis that has the property of linear phase, finite impulsive response, unitary as well as fast implementation algorithm.

In order to make wavelet basis have good frequency resolution, it should satisfies requirement of regularity:

$$
\left.\frac{d^{\prime} H_{0}(\omega)}{d \omega^{\prime}}\right|_{\omega=0}=0, l=0,1, \ldots J
$$

All Daubechies wavelets are solutions of real valued polynomial which satisfy above relation, but do not possess property of linear phase or generalized linear phase. In wavelet decomposition it inevitably introduce unwanted 
alias which decrease the coherent integration detection performance in subbands.

To obtain basis with linear phase or zero phase complex valued orthogonal transformation property, Lawton [7] considered symmetric condition, i.e. $H_{0}(z)=z H_{0}\left(z^{-1}\right)$, and constructed complex valued wavelet solutions under certain condition. Here we introduce complex symmetric orthogonal basis under general condition, through the definition of a particular polynomial introduced by Lina [8], which satisfies equation (1) and Daubechies convergent condition. It can be proved that the real symmetric Haar basis, Daubechies basis and Lawton basis are special case of the solutions of Lina polynomial. Lina polynomial is defined as:

$$
P(z)=\left(\frac{1+z}{2}\right)^{2 N+2} P_{N}\left(z^{-1}\right)
$$

where

$$
\begin{aligned}
P_{N}(z)= & \sum_{j=1}^{2 J} \alpha_{j}(z+1)^{2 N-j}(z-1)^{j}, \quad \text { and } \\
, & \left\{\begin{array}{l}
\alpha_{2 j}=(-1)^{j} 2^{-2 N} C_{2 N+1}^{j}, j=0,1, \ldots, N \\
\alpha_{2 j+1}=0
\end{array}\right.
\end{aligned}
$$

$2 J$ roots of polynomial $P_{N}(z)$ give different solution for different pasis. We can write the following factorization of $P_{N}(z)$ :

$$
H_{0}(z)=\left(\frac{1+z}{2}\right)^{1+J} \cdot q\left(z^{-1}\right)
$$

where

$q(z)=\prod_{m \in U}\left(\frac{z-x_{m}}{1-x_{m}}\right) \prod_{n \in U^{\prime}}\left(\frac{z-\overline{x_{n}}-1}{1-\overline{x_{n}}-1}\right), x_{m}, x_{n}$ are roots of $P_{N}(z)$ inside the unit circle, $\bar{x}_{n}$ is complex conjegate of $x_{n}$ and $U$ is any subsét of $\{1,2, \ldots, N\}$.

All complex valued basis functions given by Lina polynomial possess maximum flat, FIR, linear phase and fast orthogonal decomposition properties.

\section{III.DETECTION OF NLFM Signal BASED ON TIME-FREQUENCY TILING OF AWT}

Detecting under LFM signal model and using RWT to de-chirping a signal,.. in principle there lies a stationary relation between time and frequency, however, as for non-stationary property does exist in NLFM signal, it is necessary to make a joint time-frequency resolution. With the wavelet basis introduced in the previous sections, we can take advantage of the capability of arbitrary time-frequency tiling of AWT to obtain the optimal representation of non-stationary frequency variation against strong embedded. noise. Combined with fast realization algorithm, there is no need for multi-dimensional non-linear estimation and searching. Computational complexity in subband coherent processing is only subjected to one dimensional linear searching. Due to frequency variation in each tilings: possesses some relationship, it can further reduce the computational complexity of this method.

\section{A. Time frequency tiling of NLFM signals by $A W T$}

Let $\{x(n), n=1,2, \ldots N\}$ be received signal which follows NLFM model, $n$ is the discrete samples. The tree-like decomposition of $x(n)$ can be described as:

$$
\left\{\begin{array}{l}
W_{j+1,2 m}(n)=\sum_{k} W_{j, m}(k) \cdot \overline{h(k-2 n)} \\
W_{j+1,2 m+1}(n)=\sum_{k} W_{j, m}(k) \cdot \overline{g(k-2 n)}
\end{array}\right.
$$

where $W_{j, m}(n)$ denotes AWT coefficients at level $\mathrm{j}$ and subband $m, W_{j+1,2 m}(n)$ and $W_{j+1,2 m+1}(n)$ are binary tree branches of $W_{j, m}(n), h(n)$ is complex scaled filter and $g(n)$ is complex wavelet filter. Fast discrete adaptive wavelet transform can be realized by Mallat algorithm. Complex valued orthogonal filter banks make the decomposition satisfy energy conservation relationship:

$$
\|x\|^{2}=\sum_{m} \sum_{k}\left|W_{j, m}(k)\right|^{2}, m=0, \cdots, 2^{j}-1, k=0, \cdots, 2_{\xi}^{j} N-1
$$

Equation (6) means total time-frequency plane is covered with arbitrary time-frequency divisions or tilings with no redundant structure over each tilings, which ensure to remove the restored alias and obtain an optimum local representation.

An optimal representation of signal is achieved by adaptively selecting a wavepacket basis that minimizes a cost function. Bellowing are criterions to choose the cost function

a. Using a threshold $\eta$ to define a precision, computing the number of branch coefficient $W_{j, m}$ whose absolute value is greater than $\boldsymbol{\eta}$, this method is to find coefficient transferring number at certain precision.

b. For finite energy signals, choose cost function to be norm of order $P, P \in Z$, constrain the modulus of $l^{P}$ to be minimal.

c. Using variance information of branches, define $m(x)=\sum_{j} \log \left|x_{j}\right|^{2}$, to minimize $m(x)$ is equivalent to choose K-L subset of decomposition.

d. Extending Shannon entropy to define a cost function of a nil sequence, i.e. let 
$m(x)=-\sum_{k} P_{j, m}(k) \log P_{j, m}(k)$, where
$P_{j, m}(k)=\frac{\left|W_{j, m}(k)\right|^{2}}{|x|^{2}}$ is the probability of
information which satisfies $\sum_{j} \sum_{m} P_{j, m}(k)=1$, and minimal entropy means the signal energy concentration probability under certain mean error. Due to the fact that statistically distributed clutter and noise components have larger entropy, to minimize the entropy cost function is to make them diffuse in the time-frequency plane, which is helpful for coherent integration and detection in subbands.

\section{B. Time-frequency tiling based detector}

Without loss of generality, we take one NLFM signal for consideration and assume the phase of the signal be a polynomial, i.e. $x(t)=A \cdot e^{j 2 \pi \phi(t)}$, where $\varphi(t)=\sum_{i=1}^{P} a_{i} t^{i}$, and $P$ is order of polynomial phase. The detection problem can be described as:

$$
\begin{array}{ll}
H 0: & r(t)=n(t) \\
H 1: & r(t)=x(t)+n(t)
\end{array}
$$

where the $r(t)$ is the receiving signal, $n(t)$ is the i.i.d. complex Gaussian noise. Let $\Omega=\left\{P, a_{i}, i=1, \ldots, P\right\}$ be set of unknown signal parameters, we use generalized maximum likelihood estimator (GMLE) to implement an optimal receiver, i.e.

$$
\eta=\max _{\Omega}\left\{\log \left[\frac{f_{N}\left(r-A \cdot e^{j 2 \pi \sum_{i=1}^{j} \hat{a}_{i} \cdot t^{t}}\right)}{f_{N}(r)}\right]\right\}
$$

where $\hat{P}$ and $\hat{a}_{i}$ are, maximum likelihood estimation parameters of set $\Omega, f_{N}(r)$ is the $N$-dimensional joint Gaussian distribution function. Equation (8) gives the upper bound of the detector's performance, because GMLE tracks the non-linear frequency variation and performs instantaneous compensation at each samples over total time-frequency plane. But GMLE conducts a joint multi-dimensional nonlinear optimization whose computational complexity is impractical to implement. AWT based method is a sub-optimal implementation method, by using multi-resolution analysis, the method reduces the joint optimization problem of $(P+I)$ signal parameters estimation to some related one-dimensional linear searching problems which are easier to be dealt with. The detection statistic of time-frequency tiling based detector is:

$$
\eta_{A W \tau}=\sum_{m=1}^{M}\left|F\left(W_{j, m}(t) \cdot e^{-j 2 \pi \mu_{1 . t^{2}}}\right)\right|^{2}
$$

where $\mathrm{M}$ is the number of subbands, $\mu_{j, m}$ is a linear searching parameter and $F$ denotes fast Fourier transform. The detector compare $\eta_{1 W T}$ with a threshold to decide which hypothesis is true. Because there are algorithms available for LFM signal detection, so the computation complexity of the detector only depends on the size of time-frequency tiling units in principle. There is a compromise between the efficiency and the time-frequency tiling number. In many circumstances the frequency variation of a practical signal can be approached with lower-order polynomial phase model. The implementation of the proposed detector is based on the coherent integration for each tiling where the frequency variation rate is approximated to be linear. The detector is equivalent to tracking the signal time-frequency trace line by using linear approximations in each tilings while hold acceptable computation complexity.

\section{IV.NUMERICAL EXAMPLE}

To demonstrate the performance of the time-frequency tiling based detector, Monte-Carlo experiments were carried out for a NLFM signal embedded in noise. Here the performance of AWT and RWT based detectors are compared. A three-order polynomial phase signal model is used here and input signal to noise ratio is $-5 \mathrm{db}$. Both orthogonal $I$ and $Q$ dual channel complex signal are assumed to be corrupted by i.i.d. Gaussian noise.

Fig.1 illustrates the time-frequency property of signal. The instantaneous non-linear frequency variation appears to be an indistinct continuous non-linear trace line against noised background. By using complex valued adaptive wavelet transform described in the previous sections, five time-frequency subbands are achieved with each subband an almost linear variation of frequency. These subbands offer an optimal linear time-frequency approaching of signal trace line in Fig.1. Additionally, due to the time frequency tilings spread noise from one dimension into two dimensions while it concentrates signal in localized regions in time-frequency plane, SNR in subbands is higher than that in Fig.1. Fig. 2 shows these two detector outputs, in which AWT based detector has the output SNR of $11.25 d B$ while RWT based detector has that of $2.3 d B$. Fig. 3 illustrates the detection performance of two detectors. Each 1000 Monte-Carlo experiments were performed for two cases with false probability fixed at 0.01 . The results are illustrated as detection probability versus the input SNR. It can be seen from Fig. 3 that when the input SNR get lower, the AWT based detector is more robust, which is also favorable for weak NLFM signal detection. In the case of different $P d$ level, AWT detector has nearly $10 d B$ improved gain over 
RWT based one. Other simulation under different polynomial phase signal model also entrust the same conclusion, i.e., the more deviation of frequency from linearity, the better performance could be achieved, which is consisted with real situation. In another hand, the result demonstrates the good performance of the time-frequency tiling based detector.

\section{V.CONCLUSION}

In this paper, we proposed a novel time-frequency tiling based detector for detection of NLFM or PPS signal. The method exploits unitary and linear phase wavelet basis function together with joint one order linear approaching procedure to estimate the nonlinear trace line of signal in two dimensional time-frequency plane. The effectiveness of the proposed method has been confirmed by computer simulation.

\section{REFERENCES}

[1] L.Cohen, Time-frequency analysis, Englewood Cliffs, NJ, Prentice Hall,1995

[2] V.J.Kumar and C.Carral, "Performance of wigner distribution function based detection methods", Optical Engineering, Vol. 23, No. 6, 1984, 732-737.

[3] C. Wood and D.T.Barry, "Linear signal synthesis using the Radon-Wigner transform", IEEE Trans. Signal Processing, Vol. 42, No. 8, 1994, 2105-2111

[4] M.Wang, A.K.Chan and C.K.Chui, "Linear frequency modulated signal detection using Radon-ambiguity transform", IEEE Trans. Signal Processing, Vol. 46, No. $3,1998,571-586$

[5] V.C.Chen, "Applications of time-frequency processing to radar imaging", Optical Engineering, Vol. 36, No. 4, $1997,1152-1161$

[6] R.R.Coifman and M.V.Wickerhouser, "Entropy-based algorithms for best-basis selection", IEEE Trans. Inform. Theory, Vol. 38, No. 2, 1992, 713-718

[7] W.Lawton, "Applications of complex valued wavelet transforms to subband decomposition", IEEE Trans. Signal Processing, Vol. 41, No. 9, 1993, 3566-3570

[8] J. M.Lina and B.Macgibbon, "Non-linear shrinkage estimation with complex Daubechies wavelets", Wavelet Applications in Signal and Image Processing, SPIE conference, Sand Diego, 1997

[9] S.G.Mallat and Z.Zhang, "Matching pursuits with time-frequency dictionaries", IEEE Trans. Signal Processing, Vol. 41, No. 12, 1993, 3397-3415

[10]N.H.Niesen and M.V.Wickerhauser, "Wavelets and time-frequency analysis", Proceedings of the IEEE, Vol. 84 , No. 4, 1996, 523-540

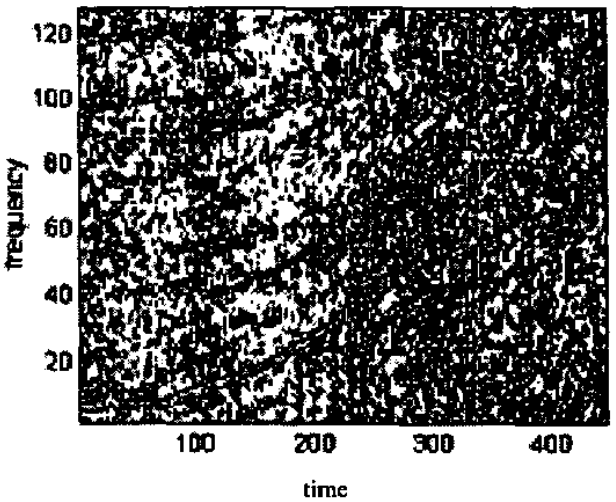

Fig. 1 Non linear time-frequency property of NLFM signal

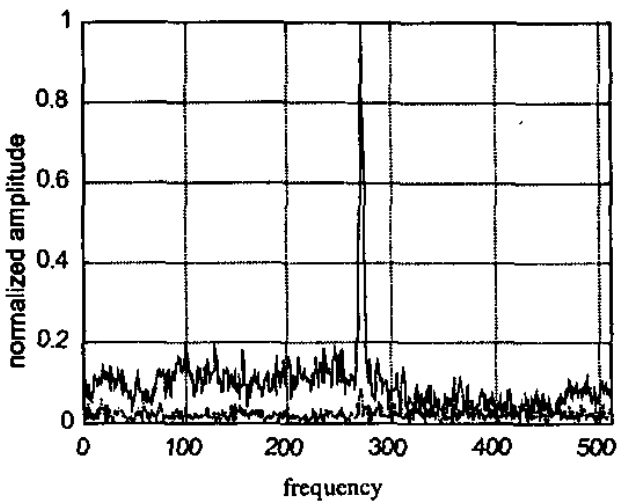

Fig. 2 Output of $t-f$ tiling and RWT based detector Solid- $t-f$ tiling based one, dash-RWT based one.

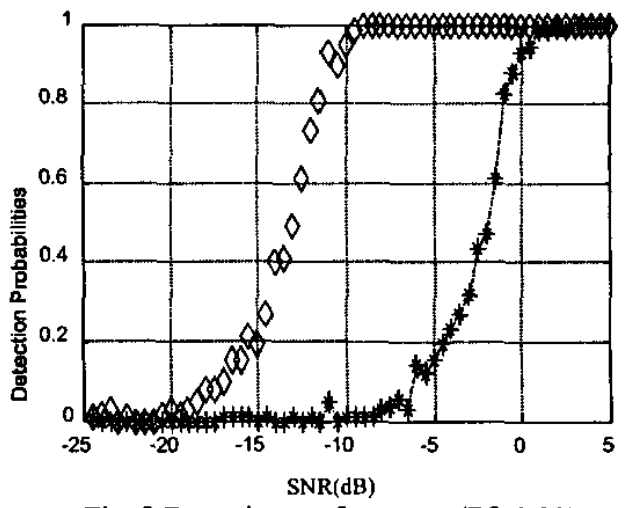

Fig. 3 Detection performance ( $\mathrm{Pf}=0.01$ )

Diamond -- $t-f$ tiling based detector, star and dash-RW'T based detector 